- Presents a case of chin trauma with a mandibular torus.

- Suggests carrying out radiographic study of the affected area to evaluate possible fracture.

- Reveals the associated presence of paraesthesia and recommends a computed tomographic study.

- No protocol treatment currently exists for this kind of fracture.

\title{
Fracture in the chin area: An unusual case of mandibular torus fracture
}

\author{
A. Saura-Ingles, ${ }^{2}$ C. Garcia-Ballesta, ${ }_{1}{ }^{\text {L. Pérez-Lajarin }}{ }^{3}$ and P. López-Jornet ${ }^{4}$
}

\begin{abstract}
Mandibular torus fracture as a result of accidental trauma has not been reported to date in the dental literature. This study describes the case of a young adult male who suffered multiple fractures affecting the teeth and mandibular torus secondary to chin area trauma due to a bicycle accident; the first manifestation of bone damage being left unilateral paraesthesia of the lip. An occlusal x-ray study of the affected area was made to evaluate possible fracture, as unlike centred periapical x-rays and orthopantomography, it is able to reveal the existence of a fracture line of the alveolar wall. In the associated presence of paraesthesia, a computed tomographic study is advisable.
\end{abstract}

\section{INTRODUCTION}

Trauma to the chin can cause violent impact of the mandibular teeth against the antagonistic upper maxillary dentition, with possible fracture of the latter via an indirect mechanism. ${ }^{1}$ Several cases have been recorded in the dental literature of severely complicated or multiple fractures of posterior teeth as a consequence of a blow to the chin area, ${ }^{2,3}$ and even mandibular and condylar fractures have been documented. ${ }^{4-7}$ Traffic accidents, bicycle falls, sports injuries and assault are all currently some of the possible mechanisms underlying blows to the maxillofacial skeleton. ${ }^{8}$

The term 'torus' refers to the existence of a convex, bony exophytic excrescence with an even base. It is a slow-growing structure (though growth may also cease spontaneously) located within the cortical bone layer. The torus core consists of spongy bone, surrounded by a dense

$1,{ }^{*} 3,4$ Assistant Professors, University of Murcia Dental School, Murcia, Spain; ${ }^{2}$ Associate Professor, University of Murcia Dental School, Murcia, Spain

${ }^{*}$ Correspondence to: Dr Pia López-Jornet, Facultad de Odontología, Hospital Morales Meseguer 2 planta. Avda, Marqués de los Vélez s/n, 30008 - Murcia, Spain Email:majornet@um.es

\section{Refereed Paper}

Received 17.09.04; Accepted 09.02.05

doi: 10.1038/sj.bdj.4812536

๑ British Dental Journal 2005; 199: 27-29

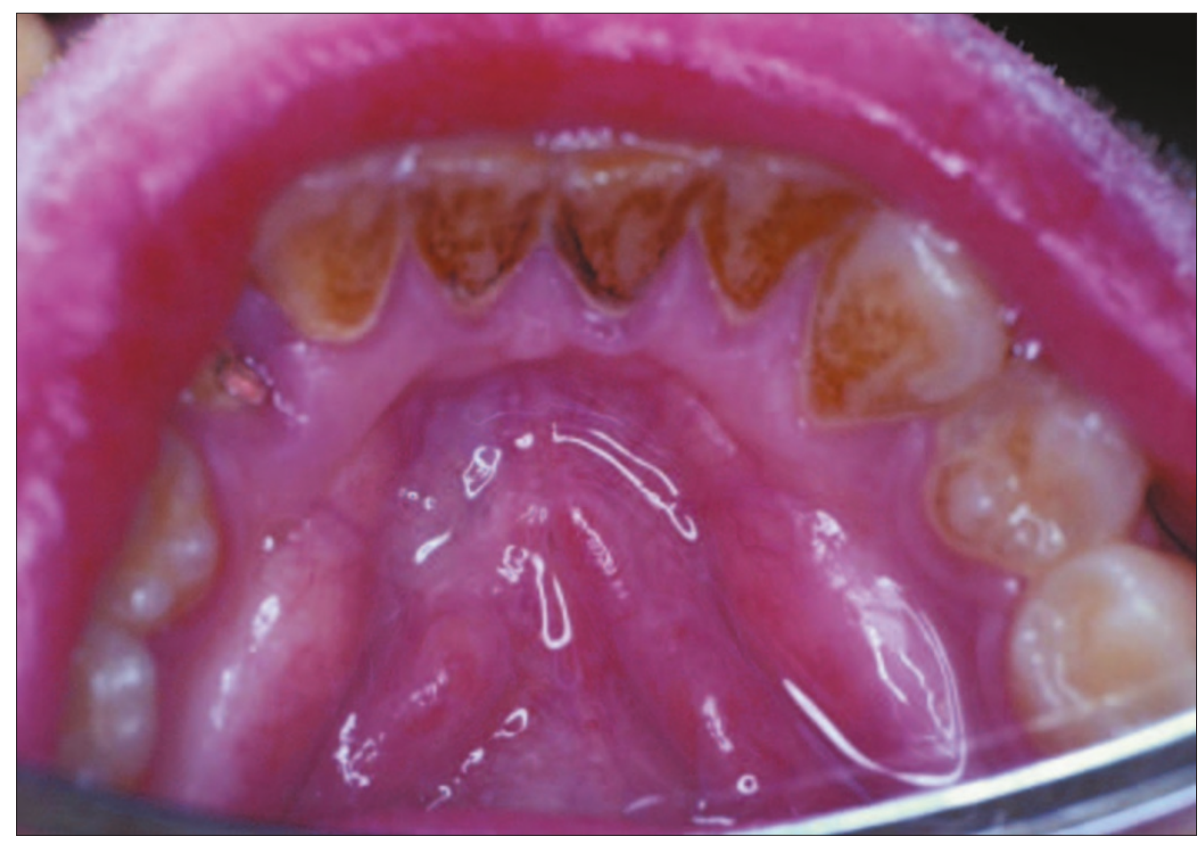

Fig. 1 Bilateral mandibular tori

cortical component. Within the lower jaw the torus is located on the lingual surface of the mandibular body, in the canine-premolar zone above the mylohyoid line. The structure can be uni- or bilateral. ${ }^{9}$

Mandibular torus fracture as a result of accidental trauma has to date not been reported in the dental literature. This study describes the case of a young adult male with multiple fractures affecting the teeth and mandibular torus secondary to chin zone trauma - the first manifestation of bone damage being unilateral paraesthesia of the lip on the left side.

\section{CLINICAL CASE}

A 26-year-old Caucasian male without antecedents of interest suffered an accident while riding a mountain bike. The patient was wearing a safety helmet, though it was not properly locked. He fell from a height of approximately 


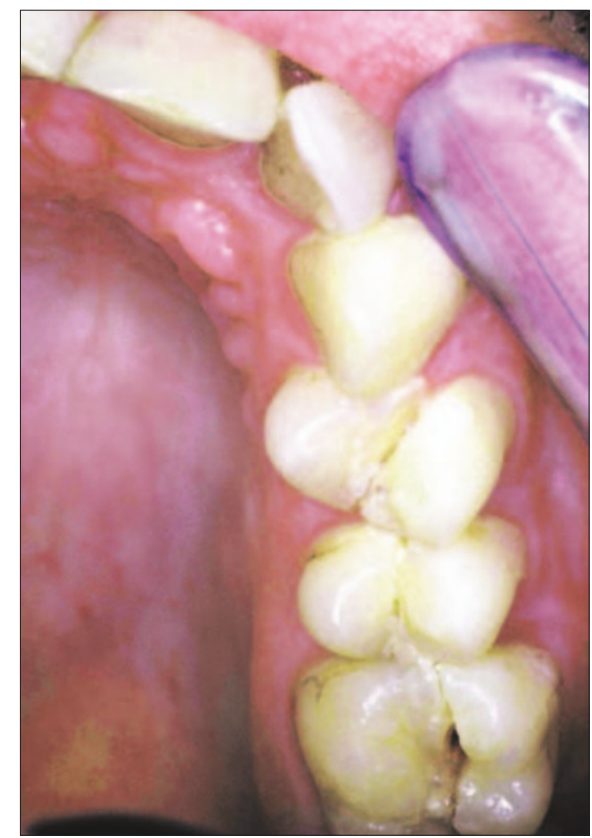

Fig. 2 The patient presented multiple complicated and uncomplicated fractures of the crown of the teeth

two metres, with interpositioning of the left forearm between the lower jaw and the ground - resulting in ulnar and carpal fracture. Following admission to hospital, neurological damage was discounted, and the patient was treated in Traumatology.

After two days the patient was referred to the dental clinic of the University of Murcia Dental School (Spain) with generalised oral pain that impeded eating. The extraoral examination revealed no lacerations or contusions in the region of the chin or parasymphyseal zone. The patient reported no pain in the condylar region, and no occlusal alterations were noted (open bite, lateral deviations or cross-bite). The intraoral study showed the presence of bilateral mandibular tori (Fig. 1) and complicated fractures of the crowns and roots of teeth $14,15,16,25$, 43 , and 47 , as well as uncomplicated fractures of the crowns of teeth 26 and 46 (Fig. 2). All fractures were confirmed by periapical radiographs. Dental management was prescribed, with endodontic treatment of 1.4, 1.6, 4.3 and 4.7, and the filling of 2.6 and 4.6. Since the crown and root fractures of 1.5 and 2.5 were longitudinal, extraction of these teeth was decided upon.

Four days after the accident and having eliminated the important dental pain, the patient reported numbness of the left side of the lip as depicted in Figure 3. Inspection revealed slight swelling below the left mandibular body and pain in response to palpation of the left half of the floor of the mouth in the region of the mandibular torus. An extraoral (orthopantomography

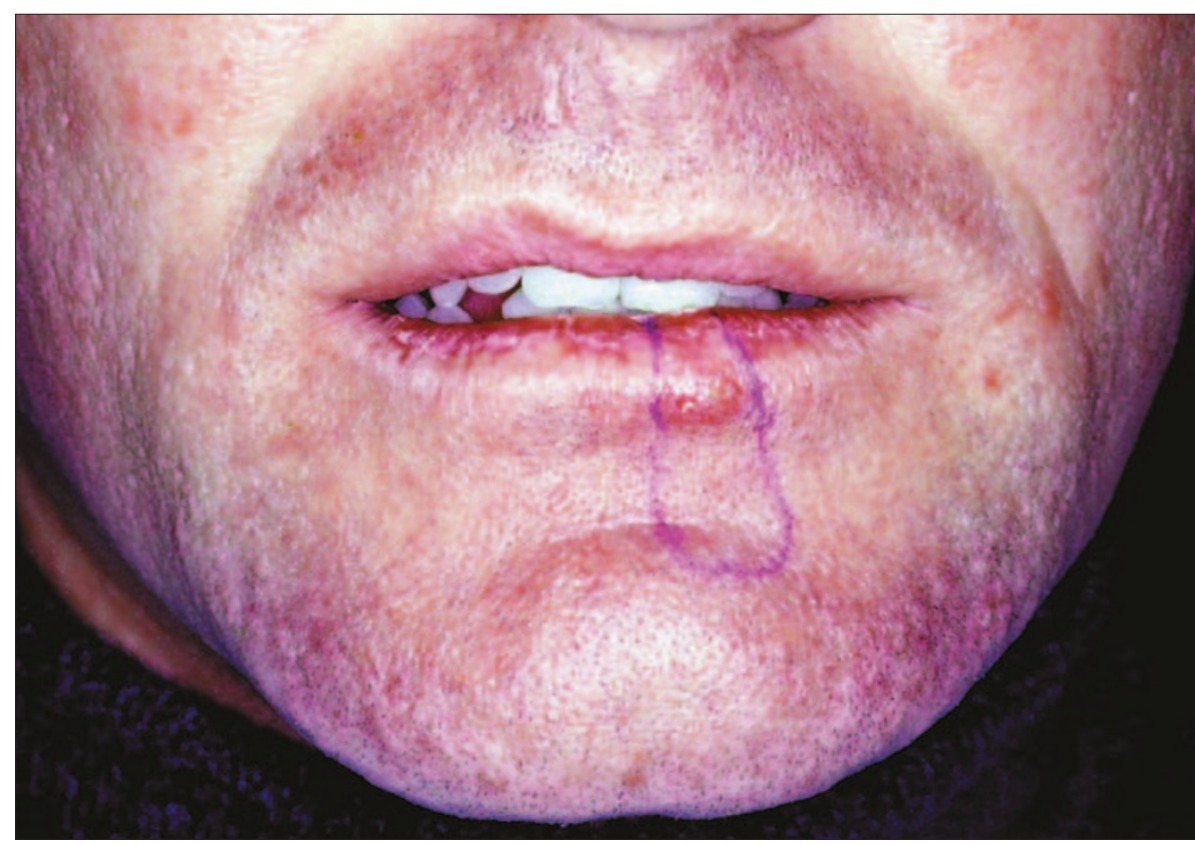

Fig. 3 Four days after the accident the patient reported numbness of the left side of the lip

and intraoral (periapical)) radiographic study revealed no abnormalities. An apical radiographic study in axial projection was then carried out (occlusal radiography), revealing a fracture line at left mandibular torus insertion level as depicted in Figure 4. A computed tomography scan confirmed the torus fracture as demonstrated in Figure 5.

After consulting Maxillofacial Surgery, patient observation was decided upon, with the prescription of ibuprofen analgesia (600 mg/8 hours). The paraesthesia gradually subsided and disappeared entirely within four weeks. A repeat computed tomography scan after six months confirmed bone consolidation as depicted in Figure 6.

\section{DISCUSSION}

Traffic accidents (50.5\%) and sports injuries (15.3\%) are currently the most common causes of facial fracture. ${ }^{8}$ In some series published in the literature (in children) bicycle falls account for up to $26 \%$ of all cases. ${ }^{10}$ The Department of Oral and Maxillofacial Surgery, University of Innsbruck, Austria, observed that mountain bikes have been found to be more frequently implicated in such injuries than conventional bicycles. ${ }^{11}$

In most situations the pathogenesis of dental trauma is not known, and little experimental information has been obtained in this field to date. ${ }^{1}$ The lesions may be secondary to either direct or indirect trauma. In this sense, indirect trauma occurs when the lower jaw violently closes upon the antagonistic teeth in the upper arch - as when a blow is delivered to the chin or mandibular corpus. This type of mechanism tends to cause dental fractures in the molar and premolar regions. ${ }^{2,3}$ Ignatius et al. ${ }^{7}$ published the frequency of dental traumas in mandibular body and condyle fractures. Dental injuries were diagnosed in $30 \%$ of cases the association with mandibular corpus fractures, and approximately 3-6 traumatised teeth per patient. Our patient presented lesions compatible with an indirect mechanism, with involvement of the posterior teeth of both arches.

Regarding bone damage, when an individual suffers a frontal fall, the neck is instinctively stretched forward to avoid direct impact to the face. ${ }^{12}$ This in turn causes the chin to be exposed to direct impact, with transmission of the kinetic energy of the blow to the mandible and temporomandibular joint. As a result, such trauma can produce fractures of the symphysis, ${ }^{1}$ condyles, ${ }^{4-6}$ glenoid fossa ${ }^{13}$ or even the cervical spine. ${ }^{14}$

The existence of a mandibular torus is not as infrequent as may be thought. In a recent study conducted in the United States, ${ }^{15} 38.7 \%$ of the individuals examined presented a torus - the latter being more common among Caucasians, and usually bilateral. In other countries, ${ }^{16}$ lower figures have been reported (5.2\% in Germany and 9.4\% in Thailand). In Spain, the reported prevalence of a mandibular torus is $7.2 \% .^{9}$

In our case, the fall could have fractured the condyles or symphysis, though the fact that the forearm buffered the impact may have reduced this possibility - increasing the likeliness of alveolar fracture, in this 


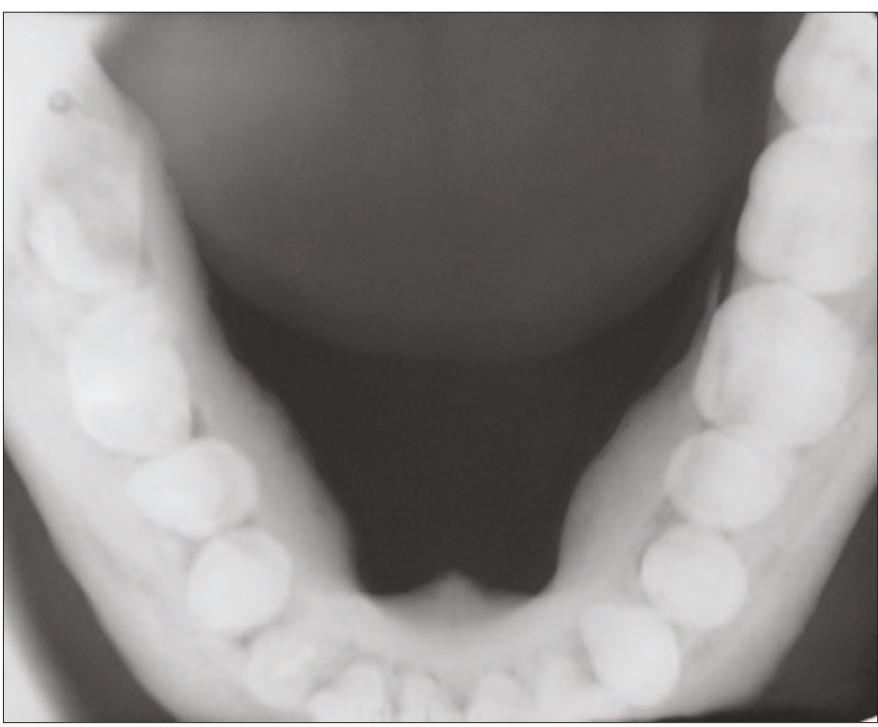

Fig. 4 Occlusal radiography revealed a fracture line at the left mandibular torus insertion level

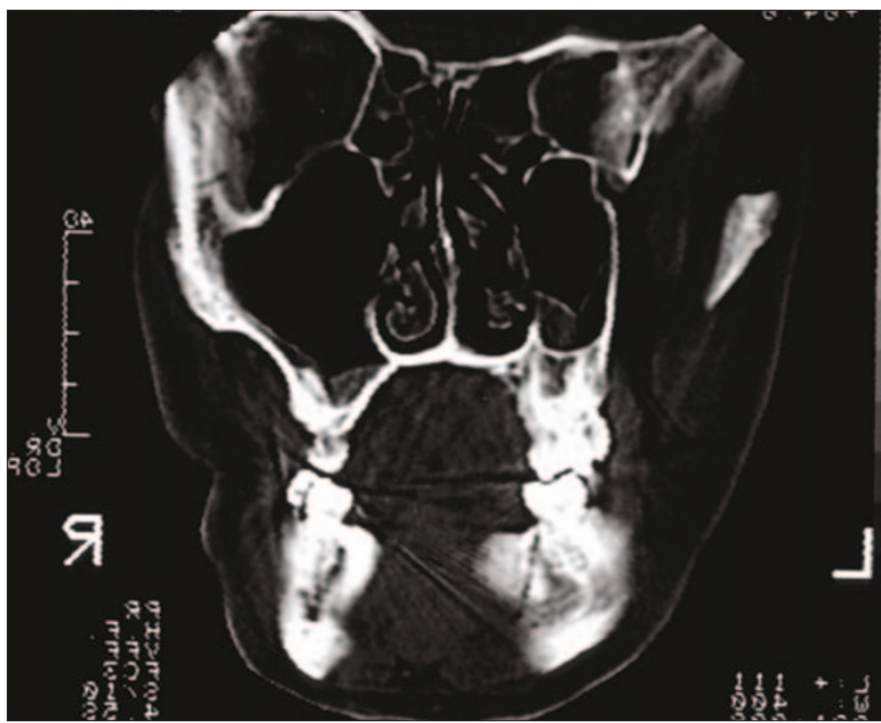

Fig. 5 A computed tomographic scan confirmed the torus fracture case of the mandibular torus. In fact, the patient suffered fracture of the forearm and carpus - a situation that would explain the absence of lacerations in the region of the chin and parasymphyseal zone. Since no cases of mandibular torus fracture secondary to trauma have been reported in the literature, we presume that the underlying mechanism was represented by impact of the forearm or wrist against the internal aspect of the mandibular body, resulting in fracture of the latter.

Regarding the transient neurological manifestations of the patient, torus fracture probably produced slight tearing of the anterior most fibres of the mental nerve, while post-trauma swelling would have compressed the nerve bundles and therefore generated paraesthesia. In any case, the damage would have been only slight, since axonal regeneration occurred within four weeks without the need of treatment. For this kind of fracture (mandibular torus) there does not yet exist a protocol treatment. ${ }^{17}$ Similar considerations are believed to apply to fracture of the genial tubercles following trauma (a conservative approach to treatment is advised with this type of fracture). ${ }^{18}$

In the case of chin trauma with the existence of a mandibular torus, we suggest conducting an occlusal radiographic study of the affected area to evaluate possible fracture, since unlike centered periapical radiography and orthopantomography, it is able to reveal the existence of a fracture line of the alveolar wall. In the associated presence of paraesthesia, a computed tomographic study is advisable.

\section{Andreasen J 0, Andreasen F M. Classification,}

etiology and epidemiology. In Textbook and color atlas of traumatic injuries to the teeth. pp 151-180. $3^{\text {rd }}$ ed. Copenhagen: Munksgaard, 1994.

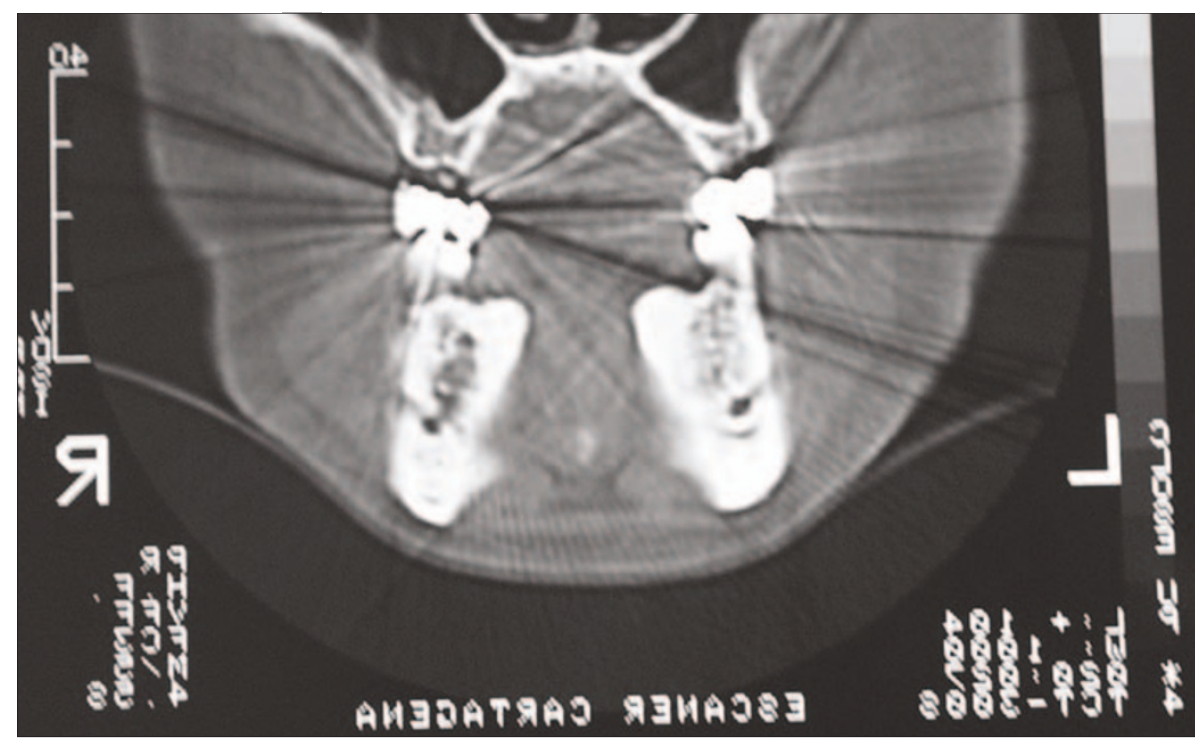

Fig. 6 A repeat computed tomography scan after six months' confirmed bone consolidation

2. Holan G. Traumatic injuries to the chin: a survey in a paediatric dental practice. Int J Paediatr Dent 1998; 8: $143-148$

3. Sasaki H, Ogawa T, Kawaguchi M, Sobue $\mathrm{S}$, Ooshima Multiple fractures of primary molars caused by injuries to the chin: report of two cases. Endod Dent Traumato/ 2000; 16: 43-46.

4. Silvennoinen U, Lindkuist C Oikarinen K. Denta injuries in association with mandibular condyle fractures. Endod Dent Traumatol 1993 9: 254-259.

5. Graziani M, Parri C, Logoluso G, Fasanelli S. Fractures of the temporomandibular joints in childhood from indirect trauma to the chin. A report of four cases. Pediatr Med Chir 1995: 17: 249-252.

6. Hurt T L, Fisher B, Peterson B M, Lynch F. Mandibular fractures in association with chin trauma in pediatric patients. Pediatr Emerg Care 1988; 4 121-123.

7. Ignatius E T, Oikarinen K, Silvennoinen U. Frequency and type of dental traumas in mandibular body and condyle fractures. Endod Dent Traumato/ 1992; 8: 235-240.

8. Van Beek G J, Merkx C A. Changes in the pattern of fractures on the maxillofacial skeleton. Int J Oral Maxillofac Surg 1999; 28: 424-428.

9. Castro Reino O R, Pérez Galera J, Pérez Cosio Martín J, Urbon Caballero J. Surgery of palatal and mandibular torus. Rev Act Odontoestomatol Esp 1990; 50: 47-56
10. lida S, Matsuya T. Paediatric maxillofacial fractures: their aetiological characters and fracture patterns. J Craniomaxillofac Surg 2002; 30: 237-241.

11. Gassner R, Tuli T, Enshoff T, and Waldart E. Mountainbiking. A dangerous sport: comparison with bicycling on oral and maxillofacial trauma. Int J Oral Maxillofac Surg 1999; 3: 188-191.

12. Profitt $W R$, Vig $K W$ L, Turvey $T A$. Early fracture of the mandibular condyles: frequently and unsuspected case of growth disturbances Am JOrthodont 1980: 78: 1-24.

13. Copenhaver R H, Dennis M J, Kloppedal E, Edwards D B, Scheffe R B. Fracture of the glenoid fossa and dislocation of the mandibular condyle into the middle cranial fossa. J Oral Maxillofac Surg 1985: 43:974-977.

14. Ardekian L, Gaspar R, Peled M, Manor R, Laufer D. Incidence and type of cervical spine injuries associated with mandibular fractures.

J Craniomaxillofac Trauma 1997; 3: 18-21.

15. Chohayeb AA, Volpe A R. Occurrence of torus palatinus and mandibularis among women of different ethnic groups. Am J Dent 2001; 14: 278-280.

16. Reichart PA, Neuhaus F, Sookasem M. Prevalence of torus palatinus and torus mandibularis in Germans and Thai. Community Dent Oral Epidemiol 1988; 16: 61-64.

17. Lazow S K. The mandible fracture: a treatment protocol. J Craniomaxillofac Trauma 1996; 2: 24-30.

18. Glendinning D E, Hirschmann P N. Fractures of the genial tubercles: two cases and a review of the literature. Br J Oral Surg 1977; 14: 217-219. 